\title{
AVALIAÇÃO DOS PRINCIPAIS ASPECTOS ASSOCIADOS AO CONSUMO DE ÁGUA NOS MUNICÍPIOS DE MINAS GERAIS E DO BRASIL
}

\author{
Brenner Henrique Maia Rodrigues ${ }^{1}$ \\ Ricardo Alexandrino Garcia ${ }^{2}$
}

\section{Resumo}

O uso da água para uso doméstico é de fundamental importância para a sociedade, sobretudo nas áreas urbanas onde o acesso a esse recurso se dá a partir de rede geral. O presente trabalho se propõe a investigar aspectos que influenciam o consumo de água doméstico no Brasil, tendo como referência dois recortes territoriais, a saber, os municípios de Minas Gerais e os municípios Brasileiros, a partir das informações registradas no Sistema Nacional de Informações de Saneamento (SNIS) para o ano de 2015. Para tanto, são aplicadas técnicas de regressão global e de regressão espacialmente ponderada $(G W R)$ a fim de determinar os parâmetros capazes de explicar o consumo de água municipal nesses dois recortes espaciais. Os modelos com melhores ajustem indicam que o consumo de água dos municípios em 2015 é explicado basicamente pela demanda (população com acesso a rede de água), pela infraestrutura existente (extensão da rede de água) e pela tarifa média praticada e que aspectos climáticos e socioeconômicos exercem pouca influência nesse consumo acumulado durante o ano.

Palavras-chave: consumo de água; abastecimento de água; saneamento básico.

\begin{abstract}
The use of water for domestic use is of fundamental importance for society, especially in urban areas where access to this resource occurs through a water network. The present work intends to investigate aspects that influence the consumption of domestic water in Brazil, having as reference two territorial cuts, namely, the municipalities of Minas Gerais and the Brazilian municipalities, based on the information registered in the National System of Sanitation Information (SNIS) for the year 2015. For this purpose, global regression and spatially weighted regression (GWR) techniques are applied in order to determine the parameters capable of explaining the municipal water consumption in these two space cuts. The best fit models indicate that municipal water consumption in 2015 is basically explained by the demand (population with access to the water network), the existing infrastructure (extension of the water network) and the average tariff practiced, and what climatic and have little influence on this consumption during the year.
\end{abstract}

Keywords: Water consumption; water supply; basic sanitation.

\footnotetext{
1 (Doutorando em Geografia pelo Programa de Pós-Graduação em Geografia - IGC/UFMG) bhmrodrigues@gmail.com

2(Professor Doutor da Universidade Federal de Minas Gerais) alexandrinogarcia@gmail.com
} 


\section{1- INTRODUÇÃO}

A água está intrinsecamente ligada ao desenvolvimento humano e tem sido essencial para os processos de crescimento urbano ao longo da história (CHAIB et al., 2015). Todavia, o crescimento do processo de urbanização no Brasil, onde a população residente em cidades passou da ordem de 10 milhões de pessoas na década de 1950 para cerca de 115 milhões em 1991 não foi acompanhado da oferta em níveis adequados dos serviços de saneamento ambiental (SANTOS, 1993; UMBELINO, 2006).

Embora a velocidade do processo de urbanização tenha reduzido a partir da década de 1990 (IBGE, 2010), a universalização dos serviços de saneamento ambiental, dentre os quais o de abastecimento de água continua sendo um desafio para o planejamento e a gestão das cidades brasileiras (GALVÃO JUNIOR, 2009). Dado esse contexto, o Brasil promulgou a lei 11.445 de cinco de janeiro de 2007 que estabeleceu diretrizes nacionais para o saneamento básico (BRASIL, 2007) cujo princípio central é a universalização dos serviços no país.

Segundo dados do último censo demográfico do Brasil $81,48 \%$ da população brasileira dispunha a época de abastecimento de água via rede geral de distribuição. No contexto urbano, o percentual da população que possui acesso a este serviço é da ordem de $91,38 \%$, enquanto na área rural corresponde a 27,79\% das pessoas (IBGE, 2010). Embora o abastecimento em áreas rurais possa demandar outras soluções tecnológica que não o abastecimento via rede geral, verifica-se que no contexto urbano ainda é grande o número de pessoas que não possui acesso a esses serviços.

Todavia, o universo descrito pelo IBGE é diferente daquele retratado pelo Sistema Nacional de Informação sobre Saneamento (SNIS), plataforma criada pelo governo federal em 1996 que contém informações e indicadores sobre a prestação de serviços de Água e Esgotos, de Manejo de Resíduos Sólidos Urbanos e Drenagem e Manejo das Águas Pluviais Urbanas fornecidas anualmente pelos prestadores desses serviços. O SNIS reúne para 2015 dados de 5079 municípios do Brasil nos quais $83,3 \%$ da população total e $93,08 \%$ da população urbana estimadas para esse ano de referência são atendidos pelo serviço de abastecimento de água dos prestadores (SNIS, 2017).

O consumo de água no Brasil acompanha ou padrão de outros países do mundo, onde o maior consumidor é o setor agrícola, responsável por cerca de $70 \%$ da demanda local, seguido pelo consumo industrial com $20 \%$ e o abastecimento doméstico com aproximadamente $10 \%$ (SETTI et al., 2001). Como a base de dados do SNIS se refere aos dados fornecidos pelos 
prestadores de serviços de saneamento, os dados retratam o consumo da água fornecida pelos mesmos (SNIS, 2017). Dessa forma, como a maior parte dos usuários dos setores agrícola e industrial capta diretamente a água, não fazem uso da água fornecida pelos prestadores de serviços de saneamento, esse consumo não compõe o objeto do presente estudo.

No Brasil, diversos trabalhos buscam analisar o consumo de água a partir de diferentes escalas. Autores como Chaib et al. (2015) e Narchi (1989) discutem os fatores que influenciam no consumo de água dentro dos domićlio, enquanto Fernandes Neto (2003), Guedes et al (2016) e Dias (2008) avaliam o consumo de água em Minas Gerais, na região nordeste e em diferentes regiões do município de Belo Horizonte (MG), respectivamente.

$\mathrm{Na}$ escala do domicílio, o consumo de água normalmente é avaliado em função de aspectos socioeconômicos, sobretudo a partir da renda e em função do tamanho dos lotes e das características físicas das edificações ocupadas pela população (CHAIB et al., 2015; DIAS, 2008). Já no contexto municipal, as análises que buscam avaliar o consumo de água discutem cinco aspectos principais, a saber, o clima, a população, a economia, o custo da água e aspectos técnicos como a pressão na rede conforme sistematizado por Von Sperling (2014).

Essas diferentes escalas de abordagem representam uma questão importante para a geografia. A noção de escala inclui tanto a relação como a inseparabilidade entre tamanho e fenômeno, conduzindo a reflexões sobre as possibilidades e os limites da correspondência de leis que regem fenômenos observados da mesma escala para fenômenos em outra escala (CASTRO, 1992; MELAZZO; CASTRO, 2007).

Dentro desse contexto, o presente trabalho se propõe a investigar aspectos que influenciam o consumo de água doméstico no Brasil, tendo como referência dois recortes territoriais, a saber, os municípios de Minas Gerais e os municípios Brasileiros, a partir das informações registradas no Sistema Nacional de Informações de Saneamento para o ano de 2015.

\section{2- METODOLOGIA}

O desenvolvimento do trabalho foi estruturado em três etapas conforme apresentado na Figura 1. A primeira etapa do trabalho consistiu na aquisição dos dados necessários ao desenvolvimento da pesquisa, sobretudo os dados associados ao abastecimento de água de 5108 municípios disponibilizados no Sistema Nacional de Informações sobre Saneamento (SNIS). Também foram obtidos dados acerca das condições acerca das condições médias de temperatura e precipitação no Brasil para o período de 1970 e 2000 (FICK; HIJMANS, 2017), do Produto 
Interno Bruto de 2014 dos municípios brasileiros (IBGE, 2014) e da Renda per capita (PNUD; IPEA; FJP, 2013).

$\mathrm{Na}$ etapa 2 foi realizado um pré-processamento dos dados e a criação de um banco de dados em ambiente de Sistema de Informações Geográficas (SIG) através do softwareArcGis 10.5 a fim de compatibilizar as informações e possibilitar a realização das análises estatísticas planejadas para a etapa subsequente. Embora o banco de dados inicial do SNIS fosse composto por dados de 5138 municípios, 39 não puderam ser analisados em função da insuficiência de dados fornecidos pelos prestadores de serviços referente ao volume de água consumido e/ou à população atendida. Dessa forma, foi criado um banco com os dados do SNIS associados à informações climáticas (precipitação e temperatura médias anuais), econômicas (PIB total e setoriais) e socioeconômica (renda per capita) que permite a realização de análises em diferentes recortes territoriais.

Já na terceira etapa do trabalho as informações sistematizadas no banco de dados foram analisadas a partir do uso de ferramentas estatísticas. Os processos foram segmentados em dois grupos, um chamado "análises estatísticas" no qual foram realizadas análises de regressão para avaliação da capacidade explicativa das variáveis analisadas e outro denominado "análises espaciais" no qual foram aplicadas ferramentas de análise espacial, a saber, o GWR e centro médios através de ferramentas implementadas no software ArcGis 10.5. Ambas as análises foram realizadas para dois recortes territoriais, a saber, os municípios do Brasil e os municípios de Minas Gerais. Os modelos gerados através desses métodos foram comparados a fim de avaliar os que apresentavam os melhores ajustes e posteriormente analisados, permitindo a discussão acerca dos fatores associados ao consumo de água. 
FigurłEstrutura metodológica da pesquisa

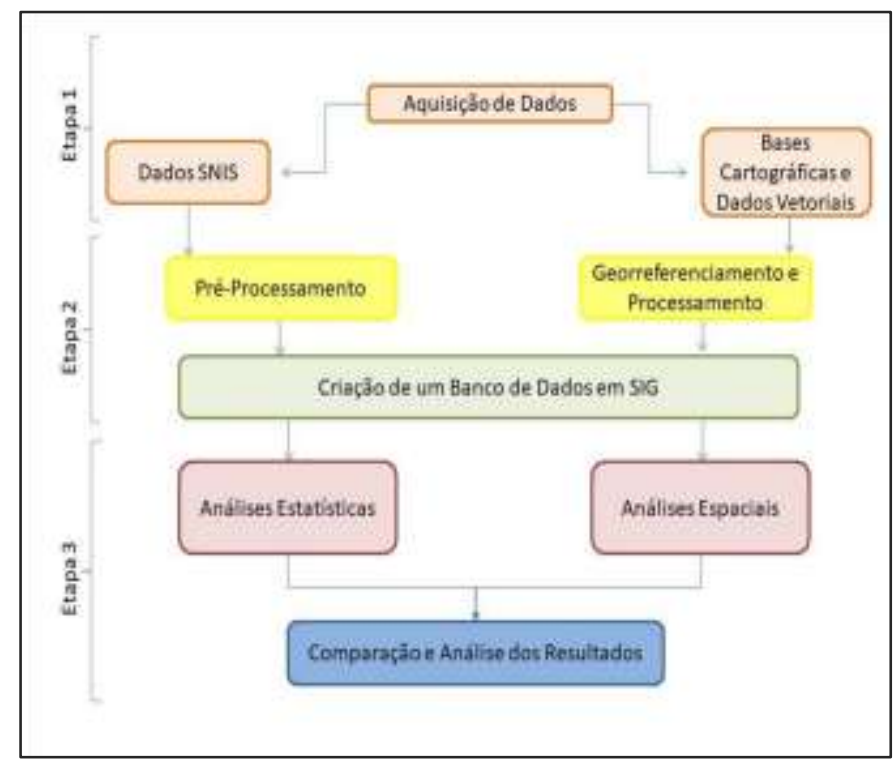

Fonte: Elaboração própria.

\section{3- CONSUMO MÉDIO DE ÁGUA NOS MUNICÍPIOS DE MINAS GERAIS}

O território mineiro, assim como todo o Brasil, apresenta grande diversidade no que se refere ao contexto físico de seu território, à dinâmica econômica e as características dos prestadores de serviços de saneamento, o que possui relação com a variação de consumo registrada em 2015. Tendo em vista os trabalhos desenvolvidos por Chaves (2016), Chaib et al, (2015), Dias (2008); Fernandes Neto (2003) e Guedes et al (1989), buscou-se avaliar a correlação entre o consumo de água dos municípios em 2015 com fatores que pudessem explica-los através de regressões lineares. Os modelos de regressão elaborados buscaram avaliar a partir de diferentes combinações o poder explicativo de variáveis associadas a infraestrutura dos serviços de abastecimento de água (número de pessoas atendidas, extensão da rede de abastecimento e tarifa média da água), às informações climáticas (precipitação e temperatura médias anuais), econômicas (PIB total e setoriais) e socioeconômica (renda per capita).

Nas regressões geradas para o conjunto dos municípios do estado de Minas Gerais as médias anuais de temperatura e de precipitação e a renda per capita tiveram pouca capacidade explicativa, enquanto a quantidade de pessoas atendidas com abastecimento de água, o PIB Municipal, a extensão da rede de água e o PIB apresentaram alto poder explicativo com $\mathrm{R}^{2}$ variando entre 0,945 e 0,983 . 
Todavia, a partir da realização de diferentes combinações entre as variáveis exploradas, verificou-se que o arranjo com o melhor poder explicativo é composto por três variáveis, a saber, população total atendida, extensão da rede de água e tarifa média da água. O resumo dos resultados dessa regressão cujo coeficiente de determinação foi de 0,985 pode ser verificado na Tabela 1.

Tabela 1: Resumo da regressão global do consumo de água nos municípios de Minas Gerais a partir da população atendida, extensão da rede de abastecimento e custo médio da tarifa em 2015.

\begin{tabular}{lr}
\hline \multicolumn{2}{c}{ Estatística de regressão } \\
\hline R múltiplo & 0,992281 \\
R-Quadrado & 0,984621 \\
R-quadrado ajustado & 0,984563 \\
Erro padrão & 710,3535 \\
Observações & 794 \\
& \\
\hline
\end{tabular}

Fonte: Elaboração própria.

Apesar dos resultados terem apresentado um bom ajuste, a utilizou-se também o modelo Geographically Weighted Regression (GWR) a fim de avaliar a predição do consumo de água nos municípios com base nos mesmos parâmetros. O GWR é uma técnica que considera a localização em sua análise de relacionamento entre as variáveis, ponderando-as em função de suas distâncias (FOTHERINGHAM; BRUNSDON; CHARLTON, 2002). Em cenários complexos como os territórios analisados, onde as variáveis relacionadas à infraestrutura e a características socioeconômicas apresentam padrões de distribuição espacial esse modelo é capaz de auxiliar na predição do consumo de água.

O uso das mesmas variáveis explicativas utilizadas na regressão global no modelo GWR gerou um $\mathrm{R}^{2}$ global ajustado de 0,992 . Verifica-se a existência de um padrão de contração espacial a partir dos valores de $\mathrm{R}^{2}$ local dos municípios do estado conforme pode ser verificado na Figura 2. Os escores que apresentam os maiores valores encontram-se concentrados na região central do estado de Minas Gerais, bem como em sua porção norte. Em contrapartida, as porções nordeste e noroeste do estado são as que apresentam os piores ajustes.

No que se refere a distribuição espacial dos resíduos padronizados (Figura3), verifica-se também que os municípios mais populosos, sobretudo aqueles concentrados na região central do estado de Minas Geris são aqueles que apresentam os maiores e menores valores. Todavia, verifica-se que 521 municípios analisados apresentam pequenos resíduos, sendo classificados na classe cujos valores variam entre -0,195 e 0,294. 
Figura 2: Regressão Espacialmente Ponderada - R2 do consumo de água nos municípios de Minas Gerais a partir da população atendida, extensão da rede de abastecimento e custo médio da tarifa em 2015.

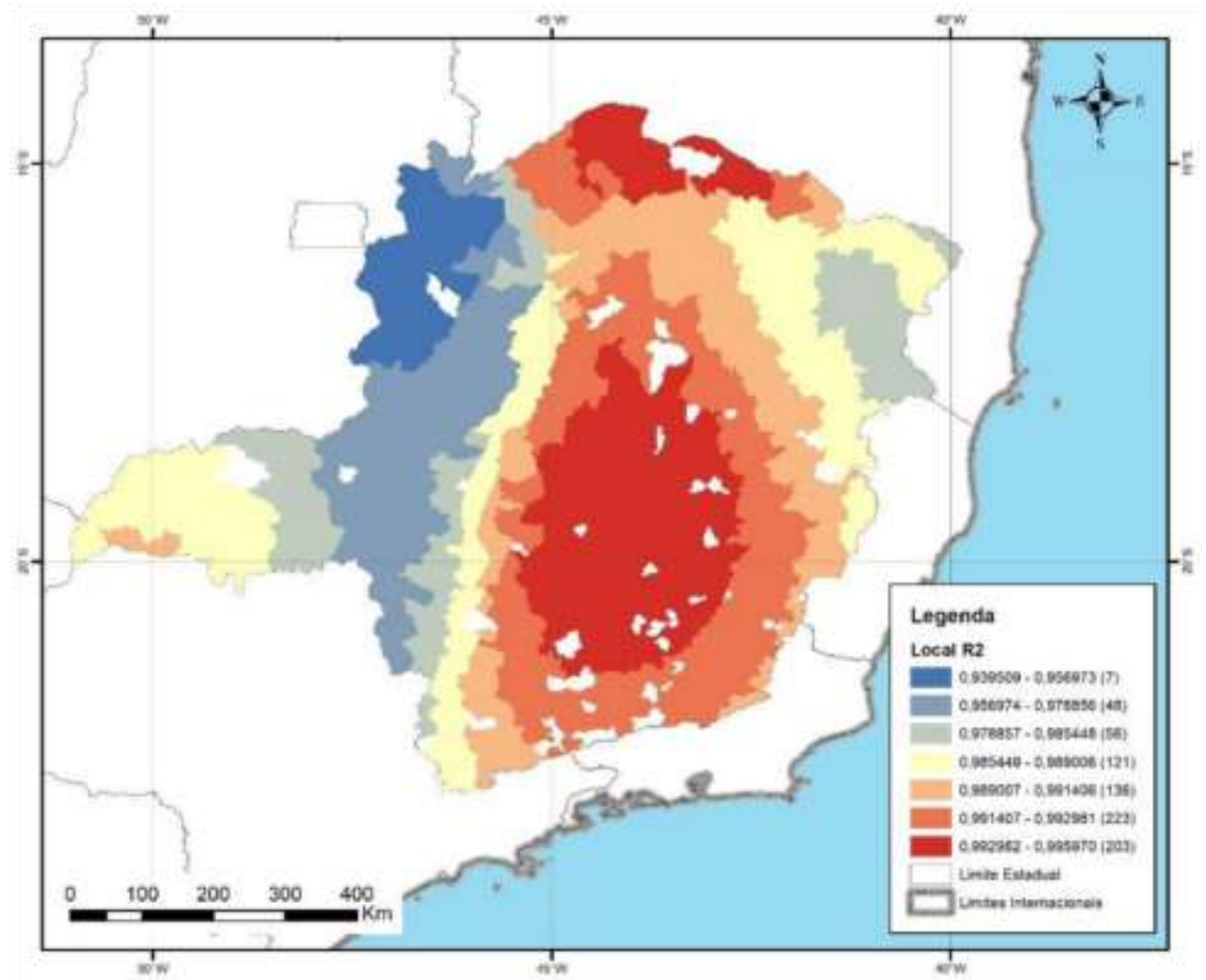

Fonte: Elaboração própria. 
Figura 3: Regressão Espacialmente Ponderada - Resíduos padronizados do consumo de água nos municípios do Brasil a partir da população atendida, extensão da rede de abastecimento e custo médio da tarifa em 2015.

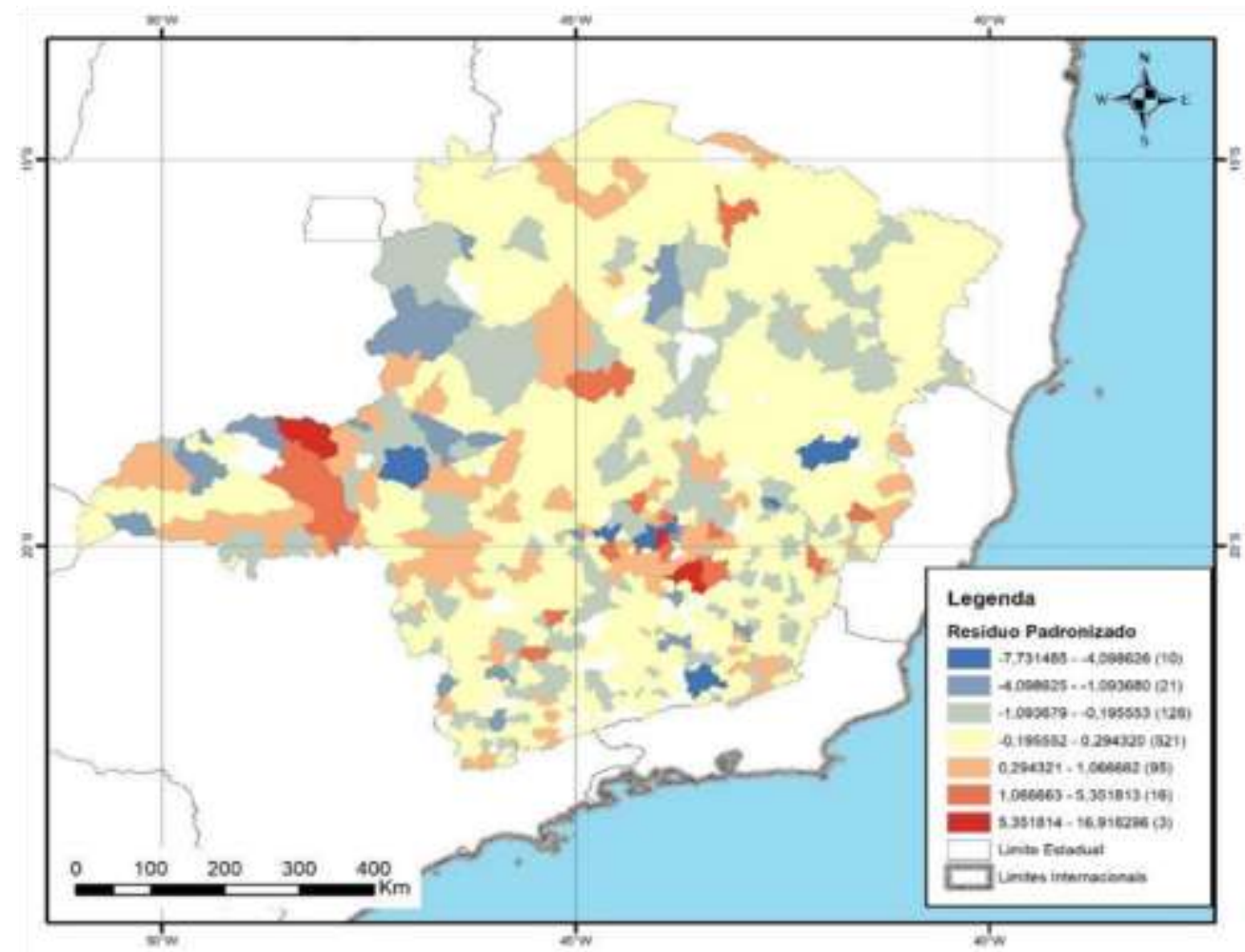

Fonte: Elaboração própria.

\section{4- CONSUMO MÉDIO DE ÁGUA NOS MUNICÍPIOS DO BRASIL}

Assim como no recorte dos municípios mineiros, buscou-se analisar os municípios do Brasil como unidade de estudo a fim de avaliar a correlação das variáveis pesquisadas com o consumo de água através de regressões lineares. Tal como ocorrido com os municípios de Minas Gerais, embora a tarifa média da água, as médias anuais de temperatura e de precipitação sejam apontadas como variáveis relacionadas ao consumo de água em domićlios e em estudos estaduais, no contexto dos municípios brasileiros essas variáveis não foram capazes de explicar individualmente o consumo de água $\left(\mathrm{R}^{2} \leq 0,001\right)$. Em contrapartida, a quantidade de pessoas atendidas com abastecimento de água, o PIB Municipal, a extensão da rede de água e a renda per capita apresentaram alto poder explicativo com $\mathrm{R}^{2}$ variando entre 0,470 e 0,859 .

Já a aplicação de regressões lineares múltiplas tendo como variáveis a população total atendida, a extensão da rede de água e a tarifa média da água, obteve como o coeficiente de determinação de 0,862 conforme pode ser verificado na Tabela 2 . 
Tabela 2: Resumo da regressão global do consumo de água nos municípios do Brasil a partir da população atendida, extensão da rede de abastecimento e custo médio da tarifa em 2015.

\begin{tabular}{lc}
\hline \multicolumn{2}{c}{ Estatística de regressão } \\
\hline R múltiplo & 0,928 \\
R-Quadrado & 0,862 \\
R-quadrado ajustado & 0,862 \\
Erro padrão & 5716,096 \\
Observações & 5099 \\
& \\
\hline
\end{tabular}

Fonte: Elaboração própria.

O uso das mesmas variáveis explicativas no modelo GWR gerou teve um $\mathrm{R}^{2}$ global ajustado de 0,936 . Os escores de $\mathrm{R}^{2}$ local da maior parte dos municípios também apresentam bom ajustes como pode ser verificado na Figura 4. Os municípios com os menores valores de $\mathrm{R}^{2}$ Local se concentram algumas regiões no sudeste do país, no centro da região sul, além dos estados do Pará e de Pernambuco.

No que se refere à distribuição dos erros padronizados, verifica-se que os erros do modelo apresentam maior concentração nos municípios mais populosos, sobretudo naqueles localizados nas regiões sudeste e sul do país (Figura 5). 
Figura 4: Regressão Espacialmente Ponderada - R2 do consumo de água nos municípios do Brasil a partir da população atendida, extensão da rede de abastecimento e custo médio da tarifa em 2015.

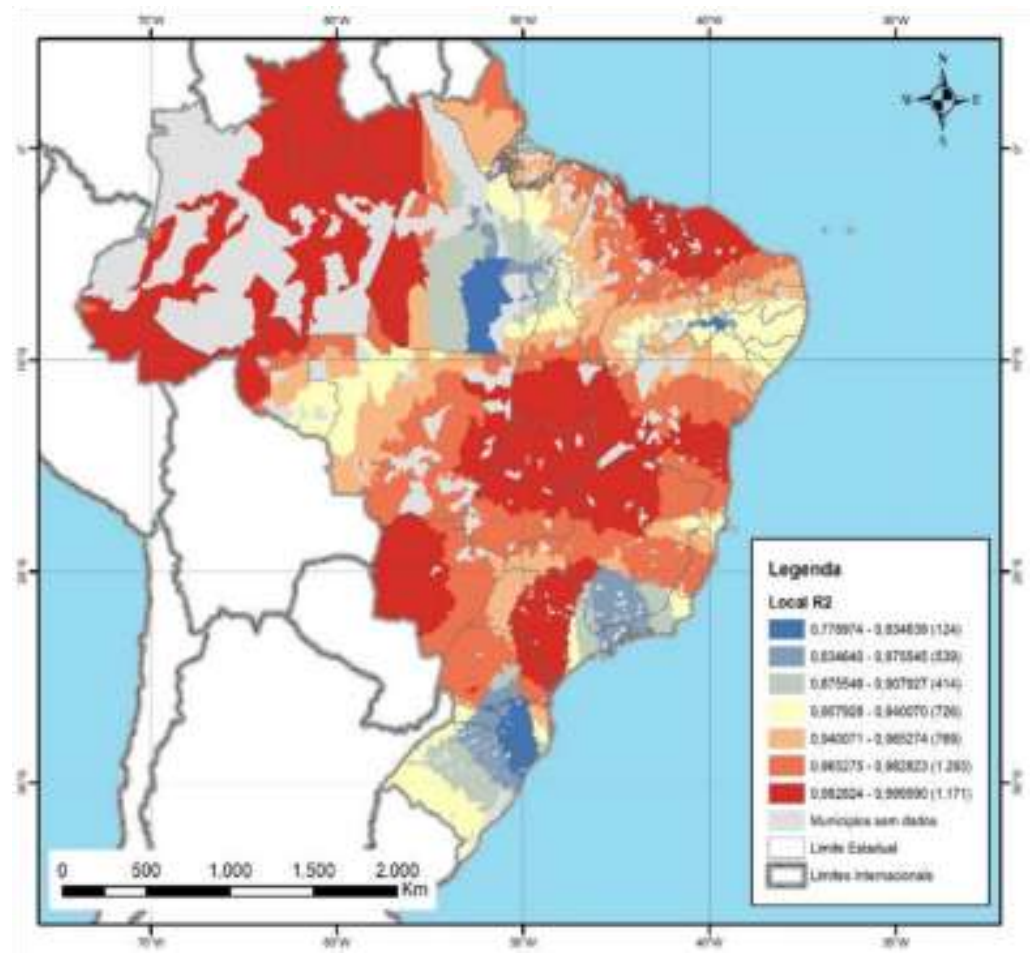

Fonte: Elaboração própria.

Figura 5: Regressão Espacialmente Ponderada - Resíduos padronizados do consumo de água nos municípios do Brasil a partir da população atendida, extensão da rede de abastecimento e custo médio da tarifa em 2015.

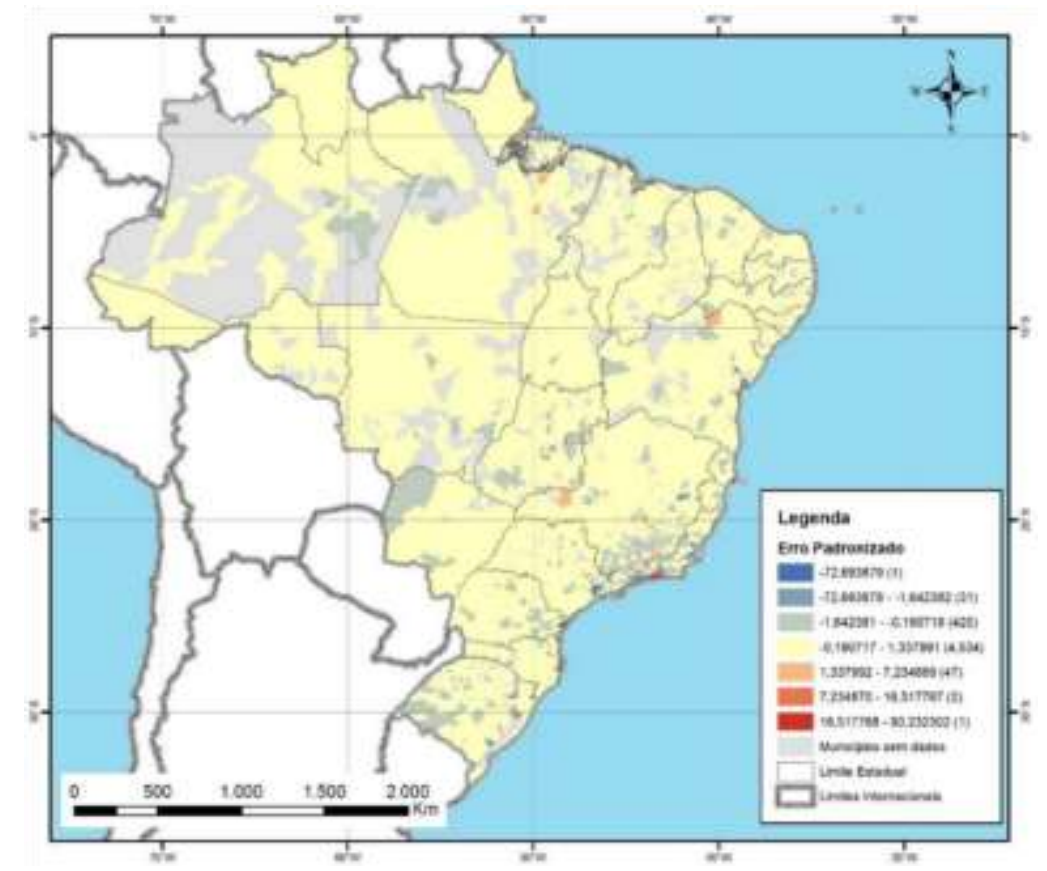

Fonte: Elaboração própria. 


\section{5- COMPARAÇÃO ENTRE OS MODELOS EM DIFERENTES ESCALAS E RECORTES TERRITORIAIS}

Embora os modelos regressivos globais tenham apresentado bons ajustes nos dois recortes espaciais utilizados, a utilizado do GWR contribui para a explicação do consumo de água. Os modelos gerados com o uso dessa técnica apresentaram altos coeficientes de determinação, indicando padrões de variabilidade espacial do consumo de água. Em ambos os casos, os melhores ajustes ocorrem na análise dos municípios do estado de Minas Gerais. Os valores dos coeficientes de determinação gerados são apresentados na Tabela 3.

Tabela 3: Comparação entre os valores de $\mathrm{R}^{2}$ nos diferentes recortes espaciais analisados.

\begin{tabular}{lcc}
\hline Recorte Territorial & $\mathbf{R}^{\mathbf{2}}$ Regressão Linear & $\mathbf{R}^{\mathbf{2}}$ GWR \\
\hline Municípios do Brasil & 0,862 & 0,936 \\
Municípios de Minas Gerais & 0,985 & 0,992 \\
\hline
\end{tabular}

Fonte: Elaboração própria.

Os resultados gerados indicam que a grande diversidade dos municípios analisado dificulta a construção de modelos para todos o território brasileiro nessa escala. Essa complexidade também dificulta a incorporação de aspectos considerados em estudos cujas escalas de análise são o domićlio para análises no contexto municipal, tal como ocorreram com as variáveis relacionadas ao clima e as condições socioeconômicas da população.

O bom ajuste dos modelos propostos para os municípios de Minas Gerais indica que a diminuição da heterogeneidade do objeto de análise pode contribuir pode contribuir para o aumento do poder explicativo dos mesmos. Embora o estado de Minas Gerais apresente heterogeneidade no que se refere aos aspectos analisados, essa complexidade tende a ser menor do que no universo dos municípios do Brasil.

\section{6- CONSIDERAÇÕES FINAIS}

A comparação entre os modelos gerados tanto através de regressões lineares, como através do GWR indicam que o número de pessoas atendidas por rede de água, a extensão dessa rede e as tarifas praticadas são fatores que explicam bem o consumo de água nos três recortes espaciais estudados. O número de pessoas atendidas por abastecimento de água é um indicador de demanda, que isoladamente já apresenta uma relação direta com o consumo de água, portanto, 
maiores concentrações demográficas tendem a consumir mais água, tanto em função do número de pessoas, como da concentração de serviços nesses locais. Esse comportamento foi verificado nos três recortes espaciais analisados.

Outro aspecto que apresentou grande correlação com o volume de água foi a extensão da rede de água, fator esse que é um indicador da infraestrutura dos serviços de saneamento nos municípios brasileiros. Como grande parte dos municípios com grandes contingentes populacionais apresenta altos níveis de verticalização, esses parâmetros contribuem para a explicação dos ajustes e dos resíduos dos modelos, sobretudo nas análises municipais.

O terceiro fator utilizado nas análises foi o custo médio da tarifa de água. Embora isoladamente esse indicador não tenha apresentado relação direta com o consumo de água, associada aos outros fatores analisados, essa variável contribuiu para a melhoria dos modelos. Verifica-se que várias das concessionárias de água, sobretudo aquelas que operam em pequenos municípios apresentam valores de tarifas muito baixos, enquanto as maiores tarifas tendem a ocorrem nos naqueles que são mais populosos.

Há que se mencionar também que as variáveis temperaturas média anual, precipitações médias anuais e o rendimento médio per capita, embora utilizadas em outros estudos que abordam o consumo de água, se mostram mais adequadas para estudos na escala de domicílios e com menores graus de agregação temporal do que para análises que envolvam municípios e estados conforme adotados no presente estudo.

\section{REFERÊNCIAS BIBLIOGRÁFICAS}

BRASIL. Lei no 11.445, de 5 de janeiro de 2007. Diário da República, Brasilia, 2007. Disponível em: <https://dre.pt/application/file/67508032>

CASTRO, I. E. DE. Analise geografica e o problema epistemológico da escala. Anuário do Instituto de Geociências, p. 21-25, 1992.

CHAIB, E. B. D. et al. Avaliação do potencial de redução do consumo de água potável por meio da implantação de sistemas de aproveitamento de água de chuva em edificações unifamiliares. RBRH - Revista Brasileira de Recursos Hídricos, v. 20, n. 3, p. 605-614, 2015.

DIAS, D. M. Avaliação do impacto da renda sobre o consumo hidrometrado de água em domicílios residenciais urbanos: Um estudo de caso para regiões de Belo Horizonte. [s.l.] Universidade Federal de Minas Gerais, 2008. 
FERNANDES NETO, M. DE L. Avaliação de parâmetros intervenientes no consumo Per capita de água: Estudo para 96 municípios do Estado de Minas Gerais. [s.l.] Universidade Federal de Minas Gerais, 2003.

FICK, S. E.; HIJMANS, R. J. Worldclim 2: New 1-km spatial resolution climate surfaces for global land areas. International Journal of Climatology, p. 1-14, 2017.

FOTHERINGHAM, A. S.; BRUNSDON, C.; CHARLTON, M. Geographically weighted regression. [s.l.] JOHN WILEY \& SONS, LTD, 2002.

GALVÃO JUNIOR, A. C. Desafios para a universalização dos serviços de água e esgoto no Brasil. RevistaPanamericana de SaludPublica/ Pan American Journal of Public Health, v. 25, n. 6, p. 548-556, 2009.

GUEDES, N. DE S.; ATHAYDE JÚNIOR, G. B.; CHAVES, G. L. R. Avaliação Do Consumo Per Capita De Água em Municípios do Nordeste do BrasilCampina GrandeAnais do VII Congressos Brasileiro de Gestão Ambiental, , 2016.

IBGE. Censo Demográfico 2010. Características da População e dos Domicílios. Instituto Brasileiro de Geografia e Estatística, p. 48, 2010.

IBGE. Produto Interno Bruto dos Municípios. 2014.

MELAZZO, E. S.; CASTRO, C. A. A escala geográfica: noção, conceito ou teoria? Terra Livre, v. 2, p. 133-142, 2007.

NARCHI, H. A demanda doméstica de água. Revista DAE, v. 49, n. 154, p. 1-7, 1989.

PNUD; IPEA; FJP. O Índice de Desenvolvimento Humano Municipal Brasileiro. Brasília: PNUD Brasil, 2013.

SANTOS, M. A urbanização Brasileira. São Paulo: Hucitec, 1993.

SETTI, A. A. et al. Introdução ao Gerenciamento de Recursos Hídricos. 2a ed ed. Brasília: ANEEL, ANA, OMM, 2001. v. 2

SNIS. Diagnóstico dos Serviços de Água e Esgotos - 2015Munistério das Cidades. Brasilia: [s.n.].

UMBELINO, G. J. DE M. Proposta metodológica para avaliação da população residente em áreas de risco ambiental: o caso da bacia hidrográfica do Córrego do Onça/MG. [s.l.] Universidade Federal de Minas Gerais, 2006.

VON SPERLING, M. et al. Investigação De Fatores De Influência No Consumo Per Capita De Água Em Estados Brasileiros E Em Cidades De Minas Gerais. VI Simpósio Ítalo Brasileiro de Engenharia Sanitária e Ambiental, p. 1-7, 2002. 
VON SPERLING, M. Introdução à qualidade das águas e ao tratamento de esgotos. 4a ed. ed. Belo Horizonte: UFMG, 2014. 